INLO-PUB-10/03

HU-EP-03/38

SFB-CPP-03-14

\title{
Axial Anomaly and Index of the Overlap Hypercube Operator
}

\author{
David H. Adams ${ }^{\mathrm{a}}$ and Wolfgang Bietenholz ${ }^{\mathrm{b}}$ \\ a Instituut-Lorentz for Theoretical Physics, Leiden University, \\ Niels Bohrweg 2, NL-2333 CA Leiden, The Netherlands \\ b Institut für Physik, Humboldt Universität zu Berlin \\ Newtonstr. 15, D-12489 Berlin, Germany
}

\begin{abstract}
The overlap hypercube fermion is constructed by inserting a lattice fermion with hypercubic couplings into the overlap formula. One obtains an exact Ginsparg-Wilson fermion, which is more complicated than the standard overlap fermion, but which has improved practical properties and is of current interest for use in numerical simulations. Here we deal with conceptual aspects of the overlap hypercube Dirac operator. Specifically, we evaluate the axial anomaly and the index, demonstrating that the correct classical continuum limit is recovered. Our derivation is non-perturbative and therefore valid in all topological sectors. At the non-perturbative level this result had previously only been shown for the standard overlap Dirac operator with Wilson kernel. The new techniques which we develop to accomplish this are of a general nature and have the potential to be extended to overlap Dirac operators with even more general kernels.
\end{abstract}

PACS: 11.15.Ha, 11.30.Rd

Keywords: Lattice fermions, Ginsparg-Wilson relation, chiral anomaly, Index Theorem

hep-lat/0307022 


\section{Introduction}

In gauge theories with fermions, the index of a Dirac operator plays an important rôle. It is given as the difference of the number of left- and right-handed zero modes, and due to the Atiyah-Singer Index Theorem [1] it can be identified with the topological charge of the gauge field. Therefore the same quantity also provides the integrated axial anomaly.

On the lattice it is a non-trivial question if these quantities can be recovered. In particular it is not clear a priori if we obtain the correct axial anomaly in the classical continuum limit. The traditional lattice formulation by means of the Wilson Dirac operator $D_{W}$ does not allow for the Index Theorem to be adapted: lattice gauge configurations do not have natural topological sectors, and the Wilson fermion does not have exact zero modes. Nevertheless the axial anomaly can be reproduced [2]; a necessary condition for this property is the absence of species doublers.

The situation is different for overlap fermions [3]. They have good chiral properties according to their origin from the overlap formalism [4, 5], which are reflected by the fact that their lattice Dirac operator $D_{o v}$ obeys the Ginsparg-Wilson relation (GWR) [6, 7]. In an even dimension $2 n$ it reads ${ }^{1}$

$$
D_{o v} \gamma_{2 n+1}+\gamma_{2 n+1} D_{o v}=\frac{a}{m} D_{o v} \gamma_{2 n+1} D_{o v}
$$

Here $m$ is a parameter which controls topological properties and the number of fermion species described by the corresponding lattice fermion action. The GWR turns into the standard condition for chiral symmetry in the continuum limit. Moreover, even on the lattice an exact chiral symmetry exists [8]. It is lattice modified by a local term of $O(a)$. Explicitly, the variation of the spinor fields under the lattice modified chiral transformation can be written in the form 8 ]

$$
\delta \bar{\psi}=\bar{\psi}\left(1-\frac{a}{2 m} D_{o v}\right) \gamma_{2 n+1}, \quad \delta \psi=\gamma_{2 n+1}\left(1-\frac{a}{2 m} D_{o v}\right) \psi,
$$

which leaves the lattice action invariant.

The Ginsparg-Wilson relation excludes additive mass renormalization. The index is well defined, since the exact zero modes have a definite chirality

\footnotetext{
${ }^{1}$ We refer to a Euclidean lattice of spacing $a$, and the fermions belong to some unitary representation of the (unspecified) gauge group.
} 
[7. ${ }^{2}$ The question arises if the correct axial anomaly is reproduced in the continuum limit. In fact, the existence of some anomaly is obvious since the fermionic measure is not invariant under the transformation (1.2). The resulting anomaly takes the form

$$
\mathcal{A}(x)=2 i q(x)
$$

where $q(x)$ is the topological charge density,

$$
q(x)=-\frac{1}{2 m} \operatorname{tr}\left(\gamma_{2 n+1} D_{o v}\right)(x, x) .
$$

Generally we define the density $\varnothing(x, y)$ of a lattice operator $\varnothing$ by

$$
\varnothing \psi(x)=a^{2 n} \sum_{y} \varnothing(x, y) \psi(y) .
$$

Then the operator trace can be expressed as

$$
\operatorname{Tr} \varnothing=a^{2 n} \sum_{x} \operatorname{tr} \varnothing(x, x)
$$

where tr sums over spinor and gauge indices. In particular the index is given by

$$
\operatorname{index}\left(D_{o v}\right)=-\frac{1}{2 m} \operatorname{Tr}\left(\gamma_{2 n+1} D_{o v}\right)=a^{2 n} \sum_{x} q(x) .
$$

The question if one obtains the correct expression for the axial anomaly has been studied in a number of papers. This issue was first addressed in Ref. 6], where a perturbative evaluation of the anomaly was given for a general Dirac operator satisfying the Ginsparg-Wilson relation. ${ }^{3}$ Furthermore, a perturbative evaluation of the axial anomaly for very general lattice Dirac operators has been carried out in Refs. [10. In all cases the anomaly was found to reduce to the correct continuum expression if the operator is local and free of species doubling. However, the use of perturbation theory restricts the validity of these arguments to the sector of topologically trivial gauge fields. In fact, the question for which Ginsparg-Wilson fermions the correct

\footnotetext{
${ }^{2}$ In the case of the overlap Dirac operator the index coincides with the overlap topological charge 4. For the fixed point fermion, which also solves the GWR, it coincides with the classically perfect topological charge 7 .

${ }^{3}$ This perturbative evaluation was reconsidered in the context of modern developments of the Ginsparg-Wilson relation [9.
} 
anomaly holds also in topological sectors different from zero seems to be non-trivial in the light of Ref. [11, which presents an example where this is apparently not the case. So far, the only Ginsparg-Wilson operator for which the correct continuum limits of the axial anomaly and index have been established non-perturbatively is the standard overlap Dirac operator, which is given by substituting the Wilson-Dirac operator $D=D_{W}$ into the overlap formula

$$
D_{o v}=\frac{m}{a}\left[1+A / \sqrt{A^{\dagger} A}\right], \quad A=D-\frac{m}{a} .
$$

The continuum limit of the axial anomaly in this case has been studied explicitly in Refs. [12, 13, 14, 15. In particular, a rigorous non-perturbative demonstration that the anomaly and the index have the correct continuum limit in all topological sectors when $m$ is in the physical (doubler-free) region was given in [13, 14].

Currently there is interest in non-standard overlap Dirac operators obtained by inserting more general lattice Dirac operators $D$ into the overlap formula (1.8). The background and motivation for this is discussed further below. In particular, overlap Dirac operators where the input $D$ is a hypercubic fermion (HF) operator have been the focus of attention. ${ }^{4}$ In the light of this development it is pertinent to show at the non-perturbative level that the axial anomaly and index for these non-standard overlap Dirac operators also have the correct continuum limit in all topological sectors. As mentioned above, this has so far only been shown for the standard overlap operator where the input $D$ is the Wilson-Dirac operator. The purpose of the present paper is to establish this result at the non-perturbative level for non-standard overlap Dirac operators of specific current interest, namely those for which the input $D$ in the overlap formula is a HF operator. To do this we follow the rigorous non-perturbative approach of Refs. [13, 14] for the standard overlap Dirac case, and take inspiration from Ref. [15, where a topological description of the anomaly coefficient as the degree of a certain map was derived which greatly facilitates its evaluation. However, the key technical parts of the arguments in those papers are specific to the standard overlap case - they rely on the explicit form of the Wilson kernel and do not have a straightforward generalization to more general kernels. Therefore we

\footnotetext{
${ }^{4} \mathrm{HF}$ operators are generalizations of the Wilson-Dirac operator which couple all sites within a lattice hypercube. (Recall that the Wilson-Dirac operator couples only nearest neighbor sites.)
} 
have had to develop new techniques and formulae to handle the more general $\mathrm{HF}$ case. In fact, our techniques are of a general nature and have the potential to be used for even more general kernels. (The case of overlap Dirac operator with completely general kernel has further complications though, and is postponed to a later analysis.)

Let us now discuss the background and motivation for considering the non-standard overlap operators mentioned above. Their use was suggested in Ref. [16. The motivation is to improve other properties of the overlap Dirac operator — beyond chirality — which are also of importance for a lattice fermion formulation, such as the quality of scaling, locality and of approximate rotation invariance. We emphasize that chiral properties of the overlap operator $D_{o v}$ continue to hold for any input lattice Dirac operator $D$ (free of species doubling) in (1.8), since $D_{o v}$ satisfies the GWR for any such choice. The basic idea is to construct a short range, doubler-free lattice Dirac operator $D$ for the input into the overlap formula which has the following properties: (i) good scaling and approximate rotational invariance, and (ii) good chirality in the sense that $D$ is an approximate solution of the GWR. The property (ii) suggests that the overlap Dirac operator $D_{o v}$ obtained from inserting $D$ into the overlap formula will inherit to a large degree the properties (i) of $D$, and will furthermore have good locality properties. To see this, note that if $D$ is an exact solution of the GWR then the overlap formula just gives $D$ back again: $D_{o v}=D$ [16]. It is known that sensible ultra-local lattice Dirac operators cannot exactly satisfy the GWR [17]; but approximate solutions are possible, and for these we have $D_{o v} \approx D$, indicating that $D_{o v}$ approximately inherits the properties of $D$ and is also likely to have good locality properties since $D$ is ultra-local.

A specific construction of a short range lattice Dirac $D$ with the properties (i) and (ii) above arises from the perfect action formalism. This formalism produces, in principle, a lattice fermion action free of artifacts in the scaling behavior via the iteration of renormalization group transformations. Moreover, the corresponding lattice Dirac operator satisfies the GWR as well 6]. The construction of the perfect action can only be carried out explicitly for free and for perturbatively interacting fermions [21. ${ }^{5}$ Still, at the nonperturbative level one can construct approximations of the classically perfect action (or fixed point action) of asymptotically free theories. The scaling

\footnotetext{
${ }^{5}$ In that case, the axial anomaly takes the correct form even at finite lattice spacing [22.
} 
artifacts of the fixed point action tend to be very small - as a study in the 2d $O(3)$ model revealed [23] - and the fixed point Dirac operator solves the GWR too [7]. Hence approximations, or truncations, of the fixed point Dirac operator are natural candidates for operators with the properties (i) and (ii) above. $^{6}$ In particular, a hypercubic approximation of the fixed point Dirac operator has been considered, and it was found in numerical studies that the use of this operator as input in the overlap formula can significantly improve the scaling, locality and convergence properties of the overlap Dirac operator 18, 19, 20]. These properties have been demonstrated in the Schwinger model [18, and also in QCD they have a potential to compensate the additional complication in $D_{H F}$ compared to $D_{W}$ : for the HF described in Ref. 19] the locality of $D_{o v}$ is improved by a factor of 2 in the exponential decay compared to the standard overlap fermion. ${ }^{7}$ Also the convergence rate increases significantly. However, to establish the overlap HF operator as a viable Dirac operator for lattice QCD one also has to check the conceptual basis, in particular whether the correct axial anomaly and index are reproduced, and this is the issue that we address in the present paper. Experiments with simpler, non-standard operators inserted in the overlap formula have been performed in Refs. [27]. All those formulations are also covered as special cases by the considerations in this paper.

The organization of the paper is as follows. In Section 2 we discuss the properties of $D_{H F}$ which are needed to compute its axial anomaly and index in Section 3. The conclusions and an outlook on further generalizations are given in the Section 4.

\footnotetext{
${ }^{6}$ For direct QCD applications of a truncated fixed point Dirac operator, see Ref. 20, 24]. However, the truncation distorts the chiral symmetry; indeed, even for truncated perfect actions the additive mass renormalization can be considerable [25]. Chiral symmetry can be re-imposed though by inserting the truncated fixed point operator into the overlap formula, and this is another motivation to study the overlap operator with truncated fixed point operator as kernel.

${ }^{7}$ This number refers to quenched $\mathrm{QCD}$ at $\beta=6$, and the corresponding test for the standard overlap fermion was performed in Ref. [26].
} 


\section{The structure of the hypercube Dirac op- erator}

We are going to use the following conventions for the $\gamma$ matrices: $\left(\gamma_{\mu}\right)^{\dagger}=$ $\gamma_{\mu},\left\{\gamma_{\mu}, \gamma_{\nu}\right\}=2 \delta_{\mu \nu}, \gamma_{2 n+1}=i^{n} \gamma_{1} \cdots \gamma_{2 n}$, so that $\left(\gamma_{2 n+1}\right)^{\dagger}=\gamma_{2 n+1}$ and $\operatorname{tr}\left(\gamma_{2 n+1} \gamma_{1} \cdots \gamma_{2 n}\right)=(-2 i)^{n}$. Throughout this paper summation over repeated indices is implied.

What we consider here is the minimally gauged HF-Dirac operator, which we are going to describe now. For techniques to simulate such HFs in QCD, see Ref. [28].

Assume the two lattice sites $x$ and $y$ to belong to the same lattice hypercube, i.e. $\left|x_{\mu}-y_{\mu}\right| \leq a$ for $\mu=1 \ldots 2 n$. Then we denote by $P(x, y)$ the set of lattice paths of minimal length connecting $x$ and $y$. All these paths are inside the same hypercube again, their length is $n_{x y} \in\{0,1, \ldots, 2 n\}$, and the number of such paths is $n_{x y}$ !. Let $\sigma$ be one of these paths. In the presence of a compact lattice gauge field $U_{\mu}(x)$ we denote by $U(\sigma)$ the product of link variables along the path $\sigma$. We also define the sign function $\epsilon(t)=\operatorname{sign} t$ for $t \neq 0$, and $\epsilon(0)=0$. Then the minimally gauged HF operator can be written as

$$
\begin{aligned}
D_{H F} & =\frac{1}{a}\left(\gamma_{\mu} \rho_{\mu}+\lambda\right) \\
\left(\rho_{\mu}\right)_{x y} & =\kappa_{n_{x y}} \frac{\epsilon\left(x_{\mu}-y_{\mu}\right)}{n_{x y} !} \sum_{\sigma \in P(x, y)} U(\sigma), \\
\lambda_{x y} & =\lambda_{n_{x y}} \frac{1}{n_{x y} !} \sum_{\sigma \in P(x, y)} U(\sigma) .
\end{aligned}
$$

The parameters $\kappa_{1}, \ldots \kappa_{2 n}$ and $\lambda_{0}, \lambda_{1}, \ldots, \lambda_{2 n}$ are coupling constants. Here we stay with the simple $\gamma$-structure of the Wilson fermion. ${ }^{8}$ The vector term $\rho_{\mu}$ alone characterizes a generalized naive fermion, which would by itself generate species doublers. The scalar term $\lambda$ can be considered a generalized Wilson term which removes these doublers (resp. attaches a mass of the cutoff scale to them) for suitable couplings, see below. $\kappa_{n_{x y}}$ and $\lambda_{n_{x y}}$ couple one site $x$ to $2^{n_{x y}}$ sites $y$. ${ }^{9}$

\footnotetext{
${ }^{8} \mathrm{HFs}$ with a more general Clifford algebra have also been used in QCD simulation, see Refs. 25, 29, 20].

${ }^{9}$ The usual Wilson-Dirac operator with Wilson parameter $r$ and bare mass $m_{0}$ is recovered by setting $\kappa_{1}=1 / 2, \lambda_{0}=2 n r+a m_{0}, \lambda_{1}=-r / 2$ and $\kappa_{j}=\lambda_{j}=0$ for $j \geq 2$.
} 
$D_{H F}$ has the correct formal continuum limit with vanishing bare mass precisely when

$$
\sum_{j=1}^{2 n} 2^{j}\left[\begin{array}{c}
2 n-1 \\
j-1
\end{array}\right] \kappa_{j}=1, \quad \sum_{j=0}^{2 n} 2^{j}\left[\begin{array}{c}
2 n \\
j
\end{array}\right] \lambda_{j}=0 .
$$

At a finite $\beta$ one deviates from these constraints and amplifies each coupling (except for $\lambda_{0}$ ) in order to compensate its suppression by the link variable [19]. However, in the current context we do impose the above constraints because they have to be restored in the classical continuum limit. They can be used to eliminate $\kappa_{1}$ and $\lambda_{0}$; then $D_{H F}$ contains $4 n-1$ free parameters $\kappa_{2}, \ldots, \kappa_{2 n} ; \lambda_{1}, \ldots, \lambda_{2 n}$.

For our purposes it is useful to express $D_{H F}$ in a coordinate-free way as follows. We use the parallel transporters

$$
T_{+\mu}(x, y)=U_{\mu}(x) \delta_{x, y-a \hat{\mu}}, \quad T_{-\mu}(x, y)=U_{\mu}^{\dagger}(x-\hat{\mu}) \delta_{x-a \hat{\mu}, y},
$$

(where $\hat{\mu}$ is the unit vector in $\mu$ direction) to define the Hermitian operators

$$
S_{\mu}=\frac{1}{2 i}\left(T_{+\mu}-T_{-\mu}\right), \quad C_{\mu}=\frac{1}{2}\left(T_{+\mu}+T_{-\mu}\right) .
$$

Moreover we introduce the following notation for the symmetrized product of operators,

$$
\left[\varnothing_{1} \ldots \varnothing_{p}\right]_{s y m}=\frac{1}{p !} \sum_{\alpha} \varnothing_{\alpha(1)} \ldots \varnothing_{\alpha(p)}
$$

where the sum runs over all permutations $\alpha$ of $\{1, \ldots, p\}$. Then eqs. (2.2) can be re-expressed as

$$
\begin{aligned}
-i \rho_{\mu} & =S_{\mu}-\sum_{p=2}^{2 n} 2^{p} \kappa_{p} \sum_{\nu_{2}<\cdots<\nu_{p} ; \nu_{j} \neq \mu \forall j}\left[S_{\mu}\left(1-C_{\nu_{2}} \cdots C_{\nu_{p}}\right)\right]_{s y m}, \\
\lambda & =\sum_{p=1}^{2 n} 2^{p}\left(-\lambda_{p}\right) \sum_{\nu_{1}<\cdots<\nu_{p}}\left[1-C_{\nu_{1}} \cdots C_{\nu_{p}}\right]_{s y m} .
\end{aligned}
$$

Note that $\rho_{\mu}^{\dagger}=-\rho_{\mu}, \lambda^{\dagger}=\lambda$, hence $D_{H F}$ satisfies $\gamma_{2 n+1}$-Hermiticity,

$$
D_{H F}^{\dagger}=\gamma_{2 n+1} D_{H F} \gamma_{2 n+1} \text {. }
$$

We now consider the zero-modes of the free field $(U=1)$ "naive" HFDirac operator $\frac{1}{a} \gamma_{\mu} \rho_{\mu}$ and their "masses" provided by the scalar term $\frac{1}{a} \lambda$ 
in eq. (2.1). The free field momentum representation of $S_{\mu}, C_{\mu}$ (i.e. their eigenvalues for the plane wave eigenfunction $e^{i k x / a}$ ) is obvious from eq. (2.5),

$$
S_{\mu}(k)=\sin \left(k_{\mu}\right) \equiv s_{\mu}, \quad C_{\mu}(k)=\cos \left(k_{\mu}\right) \equiv c_{\mu} .
$$

Hence the free field momentum representations ${ }^{10}$ of $\rho_{\mu}$ and $\lambda$ are

$$
\begin{aligned}
\rho_{\mu}(k) & =i s_{\mu}\left[1-\sum_{p=2}^{2 n} 2^{p} \kappa_{p} \sum_{\nu_{2}<\cdots<\nu_{p} ; \nu_{j} \neq \mu \forall j}\left(1-c_{\nu_{2}} \cdots c_{\nu_{p}}\right)\right] \\
\lambda(k) & =\sum_{p=1}^{2 n} 2^{p}\left(-\lambda_{p}\right) \sum_{\nu_{1}<\cdots<\nu_{p}}\left(1-c_{\nu_{1}} \cdots c_{\nu_{p}}\right)
\end{aligned}
$$

The former vanishes when $s_{\mu}=0$, so the naive HF-Dirac operator has the usual zero-mode at $k=0$ and the familiar $2^{2 n}-1$ "doubler" zero-modes for $k$ at the corners of the Brillouin zone, just as in the case of the usual naive Dirac operator. However, in addition to these, there can be other zeromodes corresponding to vanishing of the factor in the square brackets on the right-hand side of eq. (2.10). It can vanish for some momenta $k$ with components different from 0 and $\pi$, when $\kappa_{2}, \ldots, \kappa_{2 n}$ are in certain regions of the parameter space. These zero-modes correspond to new "exotic" spurious fermion species: if such a zero-mode occurs at $k=k^{(0)}$ we set $k=k^{(0)}+k^{\prime}$ and find that the leading order term in the expansion of $\gamma_{\mu} \rho_{\mu}(k)$ around $k^{(0)}$ is $\sim \gamma_{\mu} \sum_{\nu \neq \mu} k_{\nu}^{\prime}$. The corresponding propagator does not describe a usual Dirac fermion species. These exotic species are excluded though if the parameters $\kappa_{2}, \ldots, \kappa_{2 n}$ satisfy

$$
\chi_{\mu}\left[\kappa_{2}, \ldots, \kappa_{2 n}\right](k) \equiv \sum_{p=2}^{2 n} 2^{p} \kappa_{p} \sum_{\nu_{2}<\cdots<\nu_{p} ; \nu_{j} \neq \mu \forall j}\left(1-c_{\nu_{2}} \cdots c_{\nu_{p}}\right)<1 \quad \forall k .
$$

(Note that if this is satisfied for a particular index $\mu$ then it is satisfied for all $\mu=1, \ldots, 2 n$. Also, since $\chi_{\mu}=0$ at $k=0$, the condition $\chi_{\mu}<1$ is the same as $\chi_{\mu} \neq 1$.)

At this point it is natural to ask what are the values of $\kappa_{1}, \ldots, \kappa_{2 n}$ that are of interest in practice, and do they satisfy (2.12)? The values of $\lambda_{1}, \ldots, \lambda_{2 n}$ used in practice are also relevant here since they determine $\lambda(k)$ and hence

\footnotetext{
${ }^{10}$ Note that $k$ represents a re-scaled momentum, which is $2 \pi$ periodic at any lattice spacing $a$. Nevertheless we denote $k$ simply as "the momentum".
} 
the masses of the doubler fermion species. As discussed in the introduction, one of the main aims in choosing the coupling parameters is to make $D_{H F}$ as close as possible to satisfying the GW relation. The procedure of truncating perfect fermions (described in Section 1) led to the following values for the couplings [25, 16] in dimensions $2 n=2$ and $2 n=4:^{11}$

$2 n=2$ :

$$
\begin{gathered}
\kappa_{1}=0.309 \quad, \quad \kappa_{2}=0.095 \\
\lambda_{0}=1.490 \quad, \quad \lambda_{1}=-0.245 \quad, \quad \lambda_{2}=-0.128
\end{gathered}
$$

$2 n=4:$

$$
\begin{gathered}
\kappa_{1}=0.137, \quad \kappa_{2}=0.032 \quad, \quad \kappa_{3}=0.011 \quad, \quad \kappa_{4}=0.005 \\
\lambda_{0}=1.853, \lambda_{1}=-0.061, \lambda_{2}=-0.030, \lambda_{3}=-0.016, \quad \lambda_{4}=-0.008
\end{gathered}
$$

In two dimensions the left-hand side of eq. (2.12) is

$$
\chi_{\mu}^{(2 n=2)}=4 \kappa_{2}\left(1-c_{\nu}\right) \epsilon_{\mu \nu}
$$

The maximum of this, attained at $c_{\nu}=-1$, is $8 \kappa_{2}=0.76$ for the coupling values in eq. (2.13), hence eq. (2.12) is satisfied. In the dimension four case the left-hand side of eq. (2.12) can be re-written as

$$
\begin{aligned}
\chi_{\mu}^{(2 n=4)=} & 12 \kappa_{2}+24 \kappa_{3}+16 \kappa_{4}+16 \frac{\kappa_{3}^{2}}{\kappa_{2}}\left(\alpha^{3}-\left(\alpha+c_{\nu_{2}}\right)\left(\alpha+c_{\nu_{3}}\right)\left(\alpha+c_{\nu_{4}}\right)\right) \\
& -16\left(\kappa_{4}-\frac{\kappa_{3}^{2}}{\kappa_{2}}\right) c_{\nu_{2}} c_{\nu_{3}} c_{\nu_{4}}
\end{aligned}
$$

where $\left\{\mu, \nu_{2}, \nu_{3}, \nu_{4}\right\}=\{1,2,3,4\}$ and $\alpha=\kappa_{2} /\left(2 \kappa_{3}\right)$. From this we see that when $\kappa_{2} \geq 2 \kappa_{3}$ (i.e. $\alpha \geq 1$ ) and $\kappa_{4} \geq \kappa_{3}^{2} / \kappa_{2}$ the maximum of $\chi_{\mu}^{(2 n=4)}$ is attained at $c_{\nu_{2}}=c_{\nu_{3}}=c_{\nu_{4}}=-1$. For the coupling values (2.14) this maximum is 0.93 , hence (2.12) is again satisfied.

Let $\left\{k^{(j)}\right\}$ denote the momenta of the zero-modes for the free field naive HF-Dirac operator. The mass of such a mode, provided by the scalar term in $D_{H F}$, is $M^{(j)} / a$ where $M^{(j)} \equiv \lambda\left(k^{(j)}\right)$. To avoid species doubling in the full HF-Dirac operator we impose the requirement on $\lambda_{1}, \ldots, \lambda_{2 n}$ that

\footnotetext{
${ }^{11}$ We give the values to 3 decimal places; they are given to higher precision in [25, 16].
} 
$\lambda\left(k^{(j)}\right)>0$ for $k^{(j)} \neq 0$. From eq. (2.11) we see that a sufficient condition for this is $\lambda_{1}<0$ and $\lambda_{p} \leq 0 \forall p=2, \ldots, 2 n$, which holds for the coupling values in eqs. (2.13)-(2.14) (and of course also for the Wilson-Dirac operator). For the usual zero- and doubler modes, characterized by $s_{\mu}=0 \quad \forall \mu$, i.e. $k_{\mu}=0$ or $\pi$ for each $\mu$, let $N_{\pi}$ denote the number of $k_{\mu}$ 's equal to $\pi$. Then, from (2.11), the mass $M / a$ of the mode is seen to depend only on $N_{\pi}$ as

$$
M\left(N_{\pi}\right)=\sum_{p=1}^{2 n} 2^{p+1}\left(-\lambda_{p}\right) \sum_{q=1}^{p}\left[\begin{array}{c}
2 n-N_{\pi} \\
p-q
\end{array}\right]\left[\begin{array}{c}
N_{\pi} \\
q
\end{array}\right],
$$

with $\left[\begin{array}{c}N_{\pi} \\ q\end{array}\right] \equiv 0$ for $q>N_{\pi}$. From this the masses in dimensions 2 and 4 , with couplings given by eqs. (2.13) -(2.14), can be determined (see also Figures 1 and 2 in Ref. [16]). We list them in the Table below in lattice units. For comparison we also list the masses of the corresponding modes of the Wilson-Dirac operator with Wilson parameter $r$.

$2 n=2:$

\begin{tabular}{l|c|c|c|}
$N_{\pi}$ & 0 & 1 & 2 \\
\hline$M_{H F}$ & 0 & 2.004 & 1.960 \\
\hline$M_{W}$ & 0 & $2 r$ & $4 r$
\end{tabular}

$2 n=4:$

\begin{tabular}{l|c|c|c|c|c|}
$N_{\pi}$ & 0 & 1 & 2 & 3 & 4 \\
\hline$M_{H F}$ & 0 & 1.988 & 1.960 & 1.964 & 2.000 \\
\hline$M_{W}$ & 0 & $2 r$ & $4 r$ & $6 r$ & $8 r$
\end{tabular}

The HF doubler masses are all close to 2 in lattice units, reflecting the fact that the free field $D_{H F}$ with the coupling values from eq. (2.13) resp. (2.14) are good approximate solutions to the GW relation (since for exact GW solutions the eigenvalues lie on the circle in the complex plane centered at $(1 / a, 0)$ with radius $1 / a)$. We also remark that, unlike in the Wilson case, $M_{H F}$ does not always increase with increasing $N_{\pi}$.

\section{The continuum limit of the axial anomaly for the overlap-HF Dirac operator}

For a given value of the parameter $m$, the momenta of the zero-modes of the free field $D_{o v}$ are the $k^{(j)}$ with $M^{(j)}<m$ (both defined in Section 2). Hence 
the parameter region in which $D_{o v}$ has a physical zero-mode and no doublers is $0<m<\min \left\{M^{(j)} \neq 0\right\}$.

Our aim now is to evaluate the classical continuum limit of the topological charge density $q(x)$ given by eq. (1.4), or equivalently, the axial anomaly $\mathcal{A}(x)=2 i q(x)$, of the overlap-HF Dirac operator. Specifically, we consider the situation where $D_{o v}$ is coupled to the lattice transcript of a smooth continuum gauge field $A=A_{\mu}(x) d x^{\mu}$, i.e. the link variables are

$$
U_{\mu}(x)=T \exp \left(a \int_{0}^{1} A_{\mu}(x+(1-t) a \hat{\mu}) d t\right)
$$

where $T$ implies $t$-ordering. We will derive the following result:

$$
\begin{aligned}
& \text { If } m \neq M^{(j)} \quad \forall j, \text { then } \\
& \qquad \lim _{a \rightarrow 0} q(x)=I\left(\kappa_{2}, \ldots, \kappa_{2 n} ; \lambda_{1}, \ldots, \lambda_{2 n} ; m\right) q_{\text {cont }}(x)
\end{aligned}
$$

where

$$
q_{\text {cont }}(x)=\frac{1}{(2 \pi i)^{n} n !} \cdot \frac{1}{2^{n}} \epsilon_{\mu_{1} \ldots \mu_{2 n}} \operatorname{tr}\left[F_{\mu_{1} \mu_{2}}(x) \cdots F_{\mu_{2 n-1} \mu_{2 n}}(x)\right]
$$

is the continuum topological charge density and $I\left(\kappa_{2}, \ldots, \lambda_{2 n} ; m\right)$ is the degree of a certain map $\Theta: T^{2 n} \rightarrow S^{2 n}$ given in eq. (3.22) below. In particular, $I\left(\kappa_{2}, \ldots, \lambda_{2 n} ; m\right)=1$ holds for $m$ in the physical (doubler-free) region $0<$ $m<\min \left\{M^{(j)} \neq 0\right\}$. Thus, for $m$ in this region, $q(x)$ and the axial anomaly reduce to the correct continuum expressions in the classical continuum limit. Furthermore, when the parameters $\kappa_{2}, \ldots, \kappa_{2 n}$ satisfy the constraint (2.12), then

$$
I\left(\kappa_{2}, \ldots, \kappa_{2 n} ; \lambda_{1}, \ldots, \lambda_{2 n} ; m\right)=\sum_{\left\{N_{\pi}: M\left(N_{\pi}\right)<m\right\}}\left[\begin{array}{c}
2 n \\
N_{\pi}
\end{array}\right](-1)^{N_{\pi}} .
$$

We first derive the result in the infinite volume, i.e. on a hypercubic lattice on $\mathbb{R}^{2 n}$, and thereafter we discuss the finite volume $2 n$-torus case. The expression (1.4) can be re-written as

$$
q(x)=-\frac{1}{2} \operatorname{tr}\left(\frac{H_{m}}{\sqrt{H_{m}^{2}}}\right)(x, x)
$$

where

$$
H_{m}=\gamma_{2 n+1}\left(a D_{H F}-m\right)=\gamma_{2 n+1}\left(\gamma_{\mu} \rho_{\mu}+\lambda-m\right)
$$


is the Hermitian HF-Dirac operator (normalized by $1 / a$ ). We proceed as in the Wilson case treatment of Refs. [13, 14] by expanding $\left(H_{m}^{2}\right)^{-1 / 2}$ as a power series. First, $H_{m}^{2}$ is decomposed as

$$
\begin{gathered}
H_{m}^{2}=L-V \\
L=-\rho^{2}+(\lambda-m)^{2} \quad, \quad V=\gamma_{\mu}\left[\rho_{\mu}, \lambda\right]+\frac{1}{2} \gamma_{\mu} \gamma_{\nu}\left[\rho_{\mu}, \rho_{\nu}\right] .
\end{gathered}
$$

As in the Wilson case we observe $\|V\| \sim O\left(a^{2}\right)$, which is a consequence of the property $\left\|\left[T_{ \pm \mu}, T_{ \pm \nu}\right]\right\| \sim O\left(a^{2}\right)$ and eqs. (2.7). Furthermore, a lower bound $0<b<L$ exists when the lattice is sufficiently fine. ${ }^{12}$ This implies that $\left\|L^{-1} V\right\| \sim O\left(a^{2}\right)$ and consequently $\left(H_{m}^{2}\right)^{-1 / 2}=\left(L\left[1-L^{-1} V\right]\right)^{-1 / 2}$ can be expanded as a power series in $L^{-1} V$ when the lattice spacing $a$ is sufficiently small. This was done in the Wilson case [13, 14 using an integral representation of the inverse square root. (Note that the integral representation is needed since $L$ and $V$ do in general not commute.) The argument relies only on general properties of $L$ and $V$ which continue to hold in the present HF case; the treatment in Refs. [13, 14] generalizes straightforwardly to the HF and to arbitrary even dimension. Substituting the resulting expansion into eq. (3.5) and using the lattice $\delta$-function

$$
\delta_{x}=\int_{-\pi}^{\pi} \frac{d^{2 n} k}{(2 \pi)^{2 n}} e^{-i k x / a} \phi_{k}, \quad \phi_{k}(y) \equiv e^{i k y / a},
$$

to express $q(x)$ as an integral over momentum space, one obtains ${ }^{13}$

$$
q(x)=-\frac{1}{2} c(n) \frac{1}{a^{2 n}} \int_{-\pi}^{\pi} \frac{d^{2 n} k}{(2 \pi)^{2 n}} \frac{\operatorname{tr}\left(H_{m}(k) e^{-i k x / a} V^{n} e^{i k x / a}\right)}{L(k)^{n+1 / 2}}+O(a)
$$

where $H_{m}(k)$ and $L(k)$ are the free field momentum representations of $H_{m}$ and $L$, and

$$
c(n)=\left.\frac{1}{n !} \frac{d^{n}}{d t^{n}}(1-t)^{-1 / 2}\right|_{t=0}=\frac{(2 n) !}{2^{2 n}(n !)^{2}} .
$$

\footnotetext{
${ }^{12}$ This was established in the Wilson case for restricted values of $m$ in Ref. 26, 30, and later for general $m$ in 31. The result will be generalized to the present HF case, and more general cases, in 32 .

${ }^{13}$ To derive eq. (3.9) we have used the fact that the trace of the product of $\gamma_{2 n+1}$ with the product of less than $2 n \gamma$ matrices vanishes. The factor $1 / a^{2 n}$ in eq. (3.9) originates from the $a^{2 n}$ in the operator representation $\varnothing \psi(x)=a^{2 n} \sum_{y} \varnothing(x, y) \psi(y)\left(\varnothing=H_{m} / \sqrt{H_{m}^{2}}\right)$. Hence the first term is of $O(1)$.
} 
To evaluate the limit $a \rightarrow 0$ of eq. (3.9) we start from the following general observations,

$$
\begin{aligned}
& e^{-i k x / a}\left[T_{ \pm \mu}, T_{ \pm \nu}\right] e^{i k x / a}=a^{2} F_{\mu \nu}(x) e^{i\left( \pm k_{\mu} \pm k_{\nu}\right)}+O\left(a^{3}\right), \\
& e^{-i k x / a}\left[T_{ \pm \mu}, T_{\mp \nu}\right] e^{i k x / a}=-a^{2} F_{\mu \nu}(x) e^{i\left( \pm k_{\mu} \mp k_{\nu}\right)}+O\left(a^{3}\right),
\end{aligned}
$$

which imply

$$
\begin{aligned}
e^{-i k x / a}\left[S_{\mu}, S_{\nu}\right] e^{i k x / a} & =-a^{2} F_{\mu \nu}(x) c_{\mu} c_{\nu}+O\left(a^{3}\right), \\
e^{-i k x / a}\left[S_{\mu}, C_{\nu}\right] e^{i k x / a} & =a^{2} F_{\mu \nu}(x) c_{\mu} s_{\nu}+O\left(a^{3}\right) \\
e^{-i k x / a}\left[C_{\mu}, C_{\nu}\right] e^{i k x / a} & =-a^{2} F_{\mu \nu}(x) s_{\mu} s_{\nu}+O\left(a^{3}\right)
\end{aligned}
$$

with the terms defined in eq. (2.9). In the following we denote the free field momentum representation of a general lattice operator $X$ by $X(k)$. Then the relations (3.12) can be expressed collectively as

$$
e^{-i k x / a}[X, Y] e^{i k x / a}=-a^{2} F_{\alpha \beta}(x) \partial_{\alpha} X(k) \partial_{\beta} Y(k)+O\left(a^{3}\right)
$$

for $X=S_{\mu}, C_{\mu}$ and $Y=S_{\nu}, C_{\nu}$. In fact this relation continues to hold when $X$ and $Y$ are general polynomials of the $S_{\mu}$ and the $C_{\mu}$. Since $\rho_{\mu}$ and $\lambda$ are such polynomials we can apply eq. (3.13) to $e^{-i k x / a} V^{n} e^{i k x / a}$ in the expression (3.9) to obtain

$$
\begin{aligned}
& \operatorname{tr}\left(H_{m}(k) e^{-i k x / a} V^{n} e^{i k x / a}\right) \\
& =i^{n} a^{2 n} \epsilon_{\mu_{1} \ldots \mu_{2 n}} \operatorname{tr}\left(F_{\alpha_{1} \alpha_{2}}(x) \cdots F_{\alpha_{2 n-1} \alpha_{2 n}}(x)\right) \times \\
& {\left[(\lambda(k)-m) \partial_{\alpha_{1}} \rho_{\mu_{1}}(k) \cdots \partial_{\alpha_{2 n}} \rho_{\mu_{2 n}}(k)\right.} \\
& \left.\quad-2 n \partial_{\alpha_{1}} \rho_{\mu_{1}}(k) \cdots \partial_{\alpha_{2 n-1}} \rho_{\mu_{2 n-1}}(k) \partial_{\alpha_{2 n}} \lambda(k) \rho_{\mu_{2 n}}(k)\right]+O\left(a^{2 n+1}\right) .
\end{aligned}
$$

We now note the two identities, which will be crucial for our further considerations:

$$
\begin{aligned}
& \epsilon_{\mu_{1} \ldots \mu_{2 n}} \operatorname{tr}\left(F_{\alpha_{1} \alpha_{2}}(x) \cdots F_{\alpha_{2 n-1} \alpha_{2 n}}(x)\right) \partial_{\alpha_{1}} \rho_{\mu_{1}}(k) \cdots \partial_{\alpha_{2 n}} \rho_{\mu_{2 n}}(k)= \\
& \epsilon_{\mu_{1} \ldots \mu_{2 n}} \operatorname{tr}\left(F_{\mu_{1} \mu_{2}}(x) \cdots F_{\mu_{2 n-1} \mu_{2 n}}(x)\right) \epsilon_{\alpha_{1}, \ldots \alpha_{2 n}} \partial_{\alpha_{1}} \rho_{1}(k) \cdots \partial_{\alpha_{2 n}} \rho_{2 n}(k), \quad \\
& \epsilon_{\mu_{1} \ldots \mu_{2 n}} \operatorname{tr}\left(F_{\alpha_{1} \alpha_{2}}(x) \cdots F_{\alpha_{2 n-1} \alpha_{2 n}}(x)\right) \times \\
& \quad \partial_{\alpha_{1}} \rho_{\mu_{1}}(k) \cdots \partial_{\alpha_{2 n-1}} \rho_{\mu_{2 n-1}}(k) \partial_{\alpha_{2 n}} \lambda(k) \rho_{\mu_{2 n}}(k)= \\
& \epsilon_{\mu_{1} \ldots \mu_{2 n}} \operatorname{tr}\left(F_{\mu_{1} \mu_{2}}(x) \cdots F_{\mu_{2 n-1} \mu_{2 n}}(x)\right) \sum_{p=1}^{2 n}(-1)^{p} \rho_{p}(k) \epsilon_{\alpha_{0} \alpha_{1} \ldots \alpha_{p-1} \alpha_{p+1} \ldots \alpha_{2 n}} \times \\
& \partial_{\alpha_{0}} \lambda(k) \partial_{\alpha_{1}} \rho_{1}(k) \cdots \partial_{\alpha_{p-1}} \rho_{p-1}(k) \partial_{\alpha_{p+1}} \rho_{p+1}(k) \cdots \partial_{\alpha_{2 n}} \rho_{2 n}(k)
\end{aligned}
$$


These combinatorial identities rely only on the facts that the $\rho_{\mu}(k)$ and $\lambda(k)$ all commute, on $F_{\mu \nu}(x)=-F_{\nu \mu}(x)$, and on the cyclic property of the trace. Replacing the left-hand sides of these identities by the right-hand sides in eq. (3.14), and introducing the real-valued functions

$$
\theta_{0}(k)=-[\lambda(k)-m] \quad, \quad \theta_{\mu}(k)=-i \rho_{\mu}(k) \quad(\mu=1, \ldots, 2 n),
$$

we arrive at

$$
\begin{aligned}
& \operatorname{tr}\left[H_{m}(k) e^{-i k x / a} V^{n} e^{i k x / a}\right] d k_{1} \wedge \ldots \wedge d k_{2 n}= \\
& -(-i)^{n} a^{2 n} \epsilon_{\mu_{1} \ldots \mu_{2 n}} \operatorname{tr}\left(F_{\mu_{1} \mu_{2}}(x) \cdots F_{\mu_{2 n-1} \mu_{2 n}}(x)\right) \times \\
& \quad \sum_{p=0}^{2 n}(-1)^{p} \theta_{p} d \theta_{0} \wedge \ldots \wedge d \theta_{p-1} \wedge d \theta_{p+1} \wedge \ldots \wedge d \theta_{2 n}+O\left(a^{2 n+1}\right),
\end{aligned}
$$

where $d \theta_{j} \equiv \partial_{\alpha} \theta_{j} d k_{\alpha}$ is the exterior derivative of $\theta_{j}$. Substituting this into eq. (3.9) and re-writing $L$ from eq. (3.7) as

$$
L(k)=-\rho^{2}(k)+[\lambda(k)-m]^{2}=\sum_{p=0}^{2 n} \theta_{p}(k)^{2}=|\theta(k)|^{2},
$$

we obtain $\lim _{a \rightarrow 0} q(x)=I\left(\kappa_{2}, \ldots, \lambda_{2 n} ; m\right) q_{\text {cont }}(x)$, as we claimed in eq. (3.2), with

$$
\begin{aligned}
& I\left(\kappa_{2}, \ldots, \lambda_{2 n} ; m\right)=\frac{1}{2} c(n) \frac{n !}{\pi^{n}} \times \\
& \int_{[-\pi, \pi]^{2 n}} \frac{1}{|\theta|^{2 n+1}} \sum_{p=0}^{2 n}(-1)^{p} \theta_{p} d \theta_{0} \wedge \ldots \wedge d \theta_{p-1} \wedge d \theta_{p+1} \wedge \ldots \wedge d \theta_{2 n} .
\end{aligned}
$$

A little calculation shows that the integrand here can be re-written as

$$
\sum_{p=0}^{2 n}(-1)^{p} \frac{\theta_{p}}{|\theta|} d\left(\frac{\theta_{0}}{|\theta|}\right) \wedge \ldots \wedge d\left(\frac{\theta_{p-1}}{|\theta|}\right) \wedge d\left(\frac{\theta_{p+1}}{|\theta|}\right) \wedge \ldots \wedge d\left(\frac{\theta_{2 n}}{|\theta|}\right)
$$

This is precisely the pull-back to $\left.\left.T^{2 n}=\right]-\pi, \pi\right]^{2 n}$ of the volume form on the unit $2 n$-sphere $S^{2 n} \subset \mathbb{R}^{2 n+1}$ via the map

$$
\Theta: T^{2 n} \rightarrow S^{2 n} \quad, \quad \Theta(k):=\left(\frac{\theta_{0}}{|\theta|}, \frac{\theta_{1}}{|\theta|}, \ldots, \frac{\theta_{2 n}}{|\theta|}\right) .
$$


Furthermore, the coefficient of the integral in eq. (3.20) turns out to be (recall definition (3.10)

$$
\frac{1}{2} c(n) \frac{n !}{\pi^{n}}=\frac{(2 n) !}{2^{2 n+1} n ! \pi^{n}}=\frac{1}{\operatorname{Vol}\left(S^{2 n}\right)},
$$

where $\operatorname{Vol}\left(S^{2 n}\right)$ is the volume of the unit $2 n$-sphere. Hence expression (3.20) calculates the degree of the map (3.22),

$$
I\left(\kappa_{2}, \ldots, \lambda_{2 n} ; m\right)=\operatorname{deg}(\Theta)
$$

This is a generalization of the topological evaluation of the anomaly coefficient given in Ref. [15].

If $\frac{\theta}{|\theta|} \in S^{2 n} \subset \mathbb{R}^{2 n+1}$ is a regular point for the map $\Theta$ then it is a standard topological fact that $\operatorname{deg}(\Theta)=\sum_{l} s_{l}$, where $s_{l}= \pm 1$ is the sign (relative to $d k_{1} \wedge \ldots \wedge d k_{2 n}$ ) of the integrand of eq. (3.20) evaluated at a pre-image $k^{(l)}$ of $\frac{\theta}{|\theta|}$, and the sum is over all the pre-images (labelled by $l$ ). We choose $\frac{\theta}{|\theta|}=(1,0, \ldots, 0)$. Then the pre-images $k^{(l)}$ are precisely the subset of the $k^{(j)}$ introduced in Section 2 which satisfy $\theta_{0}=-[\lambda(k)-m]>0$, i.e. for which $M^{(j)}=\lambda\left(k^{(j)}\right)<m$. Moreover, the integrand in eq. 3.20 reduces at these momenta to $d \theta_{1} \wedge \ldots \wedge d \theta_{2 n}$. To determine the sign of this at a given $k^{(j)}$, recall from eq. (2.10) that $\theta_{\mu}(k)=-i \rho_{\mu}(k)=S_{\mu}(k)\left[1-\chi_{\mu}(k)\right]$ with $\chi_{\mu}(k)$ as defined in eq. (2.12). It follows that

$$
\begin{aligned}
d \theta_{1} \wedge \ldots \wedge d \theta_{2 n}= & \left(\prod_{\mu=1}^{2 n} C_{\mu}(k)\right)\left(\prod_{\nu=1}^{2 n}\left(1-\chi_{\nu}(k)\right)\right) d k_{1} \wedge \ldots \wedge d k_{2 n} \\
& + \text { terms with at least one } S_{\mu}(k) \text { factor. }
\end{aligned}
$$

If $0<m<\min \left\{M^{(j)} \neq 0\right\}$ then there is precisely one $k^{(j)}$ for which $M^{(j)}<$ $m$, namely $k^{(j)}=0$. In this case, since $\chi_{\mu}(0)=0 \forall \mu$, eq. (3.25) yields $\left.d \theta_{1} \wedge \ldots \wedge d \theta_{2 n}\right|_{k=0}=\left.d k_{1} \wedge \ldots \wedge d k_{2 n}\right|_{k=0}$, i.e. the $\operatorname{sign}$ is +1 , so $\operatorname{deg}(\Theta)=1$ in this case, as we claimed. Let us now consider the case where the parameters $\kappa_{2}, \ldots, \kappa_{2 n}$ satisfy the constraint (2.12), i.e. $\chi_{\mu}(k)<1 \forall \mu, k$. Then the $k^{(j)}$ are precisely the $k$ at which $S_{\mu}(k)=0 \forall \mu$, so the terms with $S_{\mu}(k)$ factors in eq. (3.25) vanish and the sign of the remainder is given by $\prod_{\mu=1}^{2 n} C_{\mu}\left(k^{(j)}\right)$. This sign is $(-1)^{N_{\pi}^{(j)}}$ where $N_{\pi}^{(j)}$ is the number of components of $k^{(j)}$ which are equal to $\pi$. Recalling from Section 2 that $M^{(j)}$ depends only on $N_{\pi}^{(j)}$ in this case, and noting that the number of $k^{(j)}$ with $N_{\pi}^{(j)}=N_{\pi}$ is $\left[\begin{array}{c}2 n \\ N_{\pi}\end{array}\right]$, 
it follows that $\operatorname{deg}(\Theta)=\sum_{\left\{N_{\pi}: M\left(N_{\pi}\right)<m\right\}}\left[\begin{array}{c}2 n \\ N_{\pi}\end{array}\right](-1)^{N_{\pi}}$. This completes the derivation of the claimed result (3.2)-(3.4) in the infinite lattice setting.

The rigorous derivation of the expansion (3.9), carried out along the same lines as in the overlap-Wilson case [13, 14, requires an assumption on the continuum field, namely that $A_{\mu}(x)$ and its first few partial derivatives are bounded on $\mathbb{R}^{2 n}$. Such bounds are guaranteed to exist if $A$ has a compact support on $\mathbb{R}^{2 n}$. However, having established the result in eqs. (3.2)-(3.4) for gauge fields with compact support, it can then be extended to general smooth gauge fields using locality type arguments, in the same way as in the overlap-Wilson case (see eq. (3.45) in Ref. [13] and the associated discussion). This relies on the existence of a non-zero lower bound on $H_{m}^{2}$.

In the finite volume $2 n$-torus setting the momentum integrals in eqs. (3.8), (3.9) become sums, so the derivation given above does not carry over directly to that setting. However, using locality-based arguments one can show that the finite volume $q(x)$ coincides with the infinite volume $q(x)$ up to exponentially suppressed finite size effects, thereby establishing that the continuum limit results (3.2)-(3.4) continue to hold in the finite volume setting. This was done in the overlap-Wilson case in Ref. [14; the arguments there relied only on general properties and carry over to the present HF case (given the aforementioned lower bound on $H_{m}^{2}$ ); the details of all this will be given in the general setting in Ref. 32.

In the light of the index formula (1.7) it then follows that when the overlap-HF operator is coupled to the lattice transcript of a smooth continuum gauge field $A$ on $T^{2 n}$ with topological charge $Q$, then $i n d e x\left(D_{o v}\right)$ reduces to $I\left(\kappa_{2}, \ldots, \lambda_{2 n} ; m\right) Q$ in the limit $a \rightarrow 0$. Thus the HF fermionic topological charge reduces to the continuum topological charge in the classical continuum limit when the parameter $m$ is in the doubler-free region, just as it does in the Wilson case.

\section{Summary}

We repeat that the previous literature contains the following considerations about the axial anomaly of overlap fermions:

- Perturbative considerations show that the correct continuum limit is obtained in the sector of topological charge zero for any overlap operator, see in particular Refs. [10]. 
- There was also a rigorous, non-perturbative proof that covers all topological sectors, but it was specific to the case of the simplest standard overlap fermion, which uses the Wilson-Dirac operator as an input [13, 14].

The standard overlap operator is wide-spread in recent simulations. However, there are attempts by various groups to use also non-standard overlap operators [18, 19, 20, 27. The operators used in those works are all included in the class of HF overlap operators. For the latter we have given in this paper a non-perturbative evaluation of the continuum limit of the axial anomaly and index which is valid in all topological sectors.

We have formulated the HF-Dirac operator in $2 n$-dimensional Euclidean space in the form (2.7), which is well-suited to analytic investigations. We used it first to study the dependence of the doubler structure of $D_{H F}$ on its coupling parameters. Then we evaluated the classical continuum limits of the axial anomaly and index of the overlap-HF Dirac operator, showing that the correct continuum expressions are recovered when parameters are in the physical (doubler-free) region. A noteworthy feature of our continuum limit evaluation is that it relies only on general properties of the HF-Dirac operator and not its explicit form. This is in contrast to the previous evaluations in the Wilson case (a special case of the more general HF structure) which all use the explicit form of the operator. The main new technical observations which our approach is based on are the general relation (3.13) and the identities (3.15)-(3.16). These ingredients allow the continuum form $\epsilon_{\mu_{1} \ldots \mu_{2 n}} \operatorname{tr} F_{\mu_{1} \mu_{2}}(x) \cdots F_{\mu_{2 n-1} \mu_{2 n}}(x)$ of the axial anomaly to be extracted, and its coefficient to be topologically evaluated as the degree of a map $\Theta: T^{2 n} \rightarrow S^{2 n}$ using only general properties of the HF-Dirac operator. These properties are not specific to the HF case, and the approach can be extended to completely general overlap Dirac operators obtained by substituting a general ultra-local lattice Dirac operator (involving the full Clifford algebra of $\gamma$ matrices) into the overlap formula (1.8), as it was done in Ref. [20]. The full extension, which involves considerable additional work, will be carried out in a forthcoming paper [32, and it will be shown there that the axial anomaly and index of the general overlap Dirac operator $D_{o v}$ continue to have the correct classical continuum limits in the physical (doubler-free) parameter region specified by $0<m<\min \left\{M^{(j)} \neq 0\right\}$. It was worthwhile to consider the overlap-HF case on its own, firstly because of the current interest in using this operator in numerical simulations, and secondly because 
it illustrates the main ideas and techniques of the general case but without the extensive formalism and additional complications of the latter.

Acknowledgements D.A. thanks the lattice group at Humboldt Universität, and W.B. thanks the Lorentz Instituut at Leiden University., for hospitality during visits while this work was in progress. D.A. is supported by a Marie Curie fellowship from the European Commission (contract HPMF-CT-2002-01716), and W.B. is supported in part by the DFG Sonderforschungsbereich Transregio 9, "Computergestützte Theoretische Teilchenphysik".

\section{References}

[1] M.F. Atiyah and I.M. Singer, Proc. Nat. Acad. Sci. 81 (1984) 2597.

[2] L.H. Karsten and J. Smit, Nucl. Phys B183 (1981) 103.

W. Kerler, Phys. Rev. D23 (1981) 2384.

E. Seiler and I.O. Stamatescu, Phys. Rev. D25 (1982) 2177.

N. Kawamoto and K. Shigemoto, Phys. Lett. 120B (1983) 183.

K. Fujikawa, Z. Phys. C25 (1984) 179.

H.J. Rothe and N. Sadooghi, Phys. Rev. D58 (1998) 074502.

[3] H. Neuberger, Phys. Lett. B417 (1998) 141; Phys. Lett. B427 (1998) 353.

[4] R. Narayanan and H. Neuberger, Phys. Lett. B302 (1993) 62; Phys. Rev. Lett. 71 (1993) 3251; Nucl. Phys. B412 (1994) 574; Nucl. Phys. B443 (1995) 305.

[5] S. Randjbar-Daemi and S. Strathdee, Phys. Lett. B348 (1995) 543; Nucl. Phys. B443 (1995) 386; Nucl. Phys. B466 (1996) 335; Phys. Lett. B402 (1997) 134.

[6] P.H. Ginsparg and K.G. Wilson, Phys. Rev. D25 (1982) 2649.

[7] P. Hasenfratz, V. Laliena and F. Niedermayer, Phys. Lett. B427 (1998) 125.

P. Hasenfratz, Nucl. Phys. B525 (1998) 401.

[8] M. Lüscher, Phys. Lett. B428 (1998) 342. 
[9] T.-W. Chiu and T.-H. Hsieh, hep-lat/9901011.

[10] T. Reisz and H.J. Rothe, Phys. Lett B 455 (1999) 246.

M. Frewer and H.J. Rothe, Phys. Rev. D63 (2001) 054506.

[11] T.-W. Chiu, Phys. Lett. B521 (2001) 429; Phys. Rev. D65 (2002) 054508 .

[12] Y. Kikukawa and A. Yamada, Phys. Lett. B448 (1999) 265.

K. Fujikawa, Nucl. Phys. B546 (1999) 480.

H. Suzuki, Prog. Theor. Phys. 102 (1999) 141.

[13] D.H. Adams, Ann. Phys. 296 (2002) 131.

[14] D.H. Adams, J. Math. Phys. 42 (2001) 5522.

[15] T. Fujiwara, K. Nagao and H. Suzuki, JHEP 0209 (2002) 025.

[16] W. Bietenholz, Eur. Phys. J. C6 (1999) 537.

[17] I. Horvath, Phys. Rev. Lett. 81 (1998) 4063; W. Bietenholz, hep-lat/9901005; I. Horvath, C. Balwe and R. Mendris, Nucl. Phys. B599 (2001) 283.

[18] W. Bietenholz and I. Hip, Nucl. Phys. B570 (2000) 423.

[19] W. Bietenholz, Nucl. Phys. B644 (2002) 223.

[20] P. Hasenfratz, S. Hauswirth, T. Jörg, F. Niedermayer and K. Holland, Nucl. Phys. B643 (2002) 280.

[21] W. Bietenholz and U.-J. Wiese, Nucl. Phys. B464 (1996) 319.

[22] W. Bietenholz and U.-J. Wiese, Phys. Lett. B378 (1996) 222.

[23] P. Hasenfratz and F. Niedermayer, Nucl. Phys. B414 (1994) 785.

[24] C. Gattringer et al., hep-lat/0307013.

[25] W. Bietenholz, R. Brower, S. Chandrasekharan and U.-J. Wiese, Nucl. Phys. (Proc. Suppl.) B53 (1997) 921.

[26] P. Hernández, K. Jansen and M. Lüscher, Nucl. Phys. B552 (1999) 363. 
[27] A. Boriçi, Phys. Lett. B453 (1999) 46.

T. DeGrand, Phys. Rev. D63 (2001) 034503; Phys. Rev. D64 (2001) 034512; Phys. Rev. D64 (2001) 094508; Phys. Rev. D64 (2001) 117501; Phys. Rev. D67 (2003) 014507.

T. DeGrand and A. Hasenfratz, Phys. Rev. D65 (2002) 014503.

T. DeGrand and U.M. Heller Phys. Rev. D65 (2002) 114501.

W. Kamleh, D.H. Adams, D.B. Leinweber and A.G. Williams, Phys. Rev. D66 (2002) 014501.

T. DeGrand, A. Hasenfratz and T.G. Kovacs, Phys. Rev. D67 (2003) 054501.

T.G. Kovacs, Phys. Rev. D67 (2003) 094501.

[28] W. Bietenholz, N. Eicker, A. Frommer, Th. Lippert, B. Medeke, K. Schilling and G. Weuffen, Comput. Phys. Commun. 119 (1999) 1.

[29] K. Orginos et al., Nucl. Phys. (Proc. Suppl.) B63 (1998) 904.

[30] H. Neuberger, Phys. Rev. D61 (2000) 085015.

[31] D.H. Adams, Phys. Rev. D67 (2003) 094501.

[32] D.H. Adams, work in progress. 